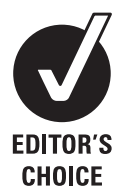

\section{${ }^{1}$ Department of}

Otolaryngology - Head and Neck Surgery, The Edith Wolfson Medical Center, Holon, Israel

Correspondence to:

Yehudah Roth

Department of Otolaryngology Head and Neck Surgery, The Edith Wolfson Medical Center, PO Box 5, 58100 Holon, Israel; orl@wolfson.health.gov.il

\title{
Limited good-quality evidence available on earwax removal methods; softeners more effective than no treatment, but evidence for irrigation or mechanical removal is equivocal
}

\section{Yehudah Roth, ${ }^{1}$ Yahav Oron, ${ }^{1}$ Abraham Goldfarb ${ }^{1}$}

\author{
Commentary on: Clegg AJ, Loveman E, Gospodarevskaya E, et al. The safety and effectiveness of \\ different methods of earwax removal: a systematic review and economic evaluation. Health Technol \\ Assess 2010;14:1-192.
}

\section{Cerumen impaction is common and saliently affects well-being}

Cerumen excreted from the skin of the external ear canal provides a protective oily layer, entraps foreign materials and has antibacterial properties. It is produced by special glands and usually is degraded in parallel, so in most people there is no wax accumulation. If a large quantity of wax is retained, it blocks the ear canal and severely interferes with hearing. Such blockage can easily be removed to resume previous hearing.

Impaction is caused by ear 'cleaning' with Q-tips which often creates a piston mechanism that pushes the wax inside the canal towards the tympanic membrane. This is one of the reasons why regular wax removal or 'cleaning' is not recommended. Some populations though tend to develop frequent cerumen impaction, which is reflected as reversible, profound, hearing loss. Other symptoms include tinnitus, itching, cough, dizziness or pain. Reasons for higher prevalence include drier secretions, metabolic changes and genetics.

Some 5\% of the general population in the UK suffers from cerumen impaction at any given time. Among the older people, prevalence reaches up to $65 \%$, and it is seen in more than $30 \%$ of the people with intellectual disability. Impaction-associated hearing impairment leads to poor communication, physical immobility, social isolation and depression. Improved cognition may follow earwax removal, and use of spray-applied new formula (Clean Ears) was superior to other common cerumenolytics. ${ }^{1}$ Old people often reconcile to hearing impairment as expected manifestation of ageing. They further tend to refrain from using assistive devices, mainly due to aesthetic considerations and financial restraints. Few residents in care homes for the older people are actually using hearing aids; the same applies to intellectually challenged people.

\section{What's new?}

The comprehensive and well-referenced review by Clegg and colleagues examines common practice and methods of cerumen removal, attempts to analyse different techniques and assumes to look into economical implications. Authors screened all relevant literature using 11 electronic resources from inception to November 2008. The study criteria included intervention methods, participants (adults/children), outcomes (hearing, wax clearance, quality of life, time to recurrence after treatment, adverse effects, cost-effectiveness) and study design. Some of the topics assessed were which agent is most effective, comparison of irrigation to mechanical removal and value of self-removal. Twenty-six clinical trials were included, with a wide variety of interventions, outcome measures, follow-up and methodological quality. The attempted analysis is admittedly difficult and not highly conclusive. Among the findings are use of cerumenolytics (cerumol, sodium bicarbonate, olive oil, water, triethanolamine polypeptide) is more effective than no treatment; sodium bicarbonate drops followed by irrigation by nurse is more effective than drops followed by self-irrigation; endoscopic de-waxing is better than microscopic de-waxing. Adverse effects appeared to be minor and of limited extent. Softeners followed by self-irrigation were more likely to be cost effective than softeners followed by irrigation at primary care. The authors conclude that some measure of treatment is better than no treatment at all, that the question of which softener is most effective is still open and that evidence is equivocal as to the effectiveness of irrigation versus mechanical removal.

Despite their elegant attempts to put some order into the diverse data, the authors found it difficult to differentiate between the various methods and regard their own economic evaluation as speculative. At best, they could assume that this procedure may be costeffective, we concur. The strongest evidence-based justification is the illuminating smile of an old woman who enters our clinic practically deaf and walks out after 2 minutes on her way to attend a lecture at the local community centre.

\section{Clearing the cerumen - the mission}

Facing an old or intellectually challenged person with hearing deterioration, it is the nurse's role to consider the possibility that this person has a wax-associated reversible hearing loss which can be easily verified by a simple otoscopy and corrected within a few minutes or up to 1 week.

Removal of cerumen should be preceded by the use of a cerumenolytic agent. The clearing itself, regardless of the chosen technique, should be conducted cautiously by qualified and experienced medical personnel to avoid ear canal or tympanic membrane injury or infection. Clearing should be conducted with the goal of improving hearing and not as an attempt to remove all the cerumen from the ear.

In Israel, a number of surveys conducted at homes for the older people and for people with intellectual disability revealed a high rate of wax impaction and demonstrated the feasibility of rapid and effective mass treatment, using 
minimal equipment and staffing. It was estimated that an institution of 100 residents would usually require just two visits of a small team, each visit lasting a full working day. Following these surveys, the national medical guidelines of the respective government agencies were updated, and a comprehensive awareness and training programme was initiated.
Competing interests None.

\section{Reference}

1. Oron Y, Zwecker-Lazar I, Levy D, et al. Cerumen removal: comparison of cerumenolytic agents and effect on cognition among the elderly. Arch Gerontol Geriatr 2011;52:228-32. 